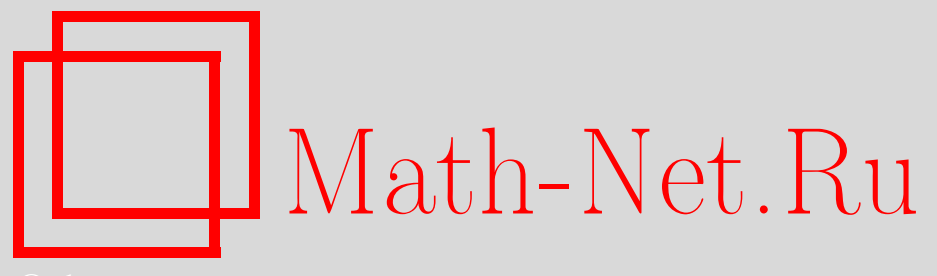

М. Н. Саушкин, А. Ю. Куров, Анализ напряжённого состояния в надрезах полукруглого профиля после опережающего поверхностного пластического деформирования сплошных цилиндрических образцов, Вестн. Сам. гос. техн. ун-та. Сер. Физ.-мат. науки, 2012, выпуск 1(), 133-140

DOI: https://doi.org/10.14498/vsgtu1039

Использование Общероссийского математического портала Math-Net.Ru подразумевает, что вы прочитали и согласны с пользовательским соглашением

http://www.mathnet.ru/rus/agreement

Параметры загрузки:

IP : 54.84 .234 .179

26 апреля 2023 г., 14:36:22

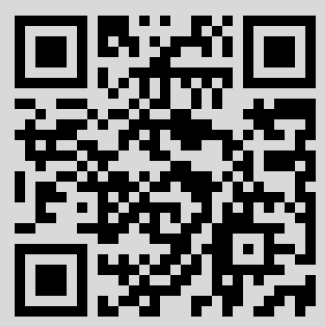


Вестн. Сам. гос. техн. ун-та. Сер. Физ.-мат. науки. 2012. № 1 (26). С. $133-140$

УДК 539.43:621.787

\title{
АНАЛИЗ НАПРЯЖЁННОГО СОСТОЯНИЯ В НАДРЕЗАХ ПОЛУКРУГЛОГО ПРОФИЛЯ ПОСЛЕ ОПЕРЕЖАЮЩЕГО ПОВЕРХНОСТНОГО ПЛАСТИЧЕСКОГО ДЕФОРМИРОВАНИЯ СПЛОШНЫХ ЦИЛИНДРИЧЕСКИХ ОБРАЗЦОВ
}

\author{
М. Н. Саушкин, А. Ю. Куров \\ Самарский государственный технический университет, \\ 443100, Россия, Самара, ул. Молодогвардейская, 244. \\ E-mails: msaushkin@gmail.com, alexeykurov@gmail.com
}

\begin{abstract}
Исследуется распределение остаточных напряжений в сплошных поверхностно упрочнённых иилиндрических образцах и образцах с полукруглым надрезом с использованием метода конечных элементов. В качестве исходной информаиии используется одна и/или две экспериментально определённые компоненты остаточных напряжений в упрочнённом слое. Отмечено, что на перераспределение остаточных напряжений в области концентратора напряжений существенное влияние оказывает отношение радиуса надреза к глубине обнуления осевой компоненты остаточных напряэений.
\end{abstract}

Ключевые слова: распределение остаточных напряжений, иилиндрический образеи, полукруглый надрез, опережсающее упрочнение, метод конечных элементов.

Хорошо известно, что зоны концентрации напряжений в деталях машин и элементах конструкций являются очагами зарождения усталостных трещин и хрупких разрушений, развитие которых приводит в конечном итоге к их разрушению. Результаты многих исследователей свидетельствуют о положительном влиянии остаточных напряжений на выносливость изделий с концентраторами напряжений, поэтому для увеличения сопротивления усталости таких изделий в местах, где может произойти разрушение, при их изготовлении наводят сжимающие остаточные напряжения, как правило, методами поверхностного пластического деформирования.

Упрочнение деталей, имеющих на поверхностях концентраторы напряжений, может производиться после их изготовления, если материал детали допускает и хорошо воспринимает пластическое деформирование поверхностного слоя на глубину, превышающую соответствующие размеры концентраторов. В противном случае, когда размеры концентратора малы или доступ к нему ограничен, поле сжимающих остаточных напряжений в зоне концентратора напряжений стараются наводить при изготовлении. Иногда на практике изготовлению мелких концентраторов напряжений предшествует упрочнение гладкой детали (опережающее упрочнение поверхности детали). При таком подходе после упрочнения в детали возникает неоднородное поле остаточных пластических деформаций и неоднородное по глубине залегания поле остаточных напряжений, а после изготовления концентратора напряжений (фактически - удаления части объёма) под действием остаточных пластических деформаций, играющих роль начальных деформаций, в упрочнённом

Саушкин Михаил Николаевич (к.ф.-м.н., доц.), докторант, каф. прикладной математики и информатики. Алексей Юръевич Куров, студент, каф. прикладной математики и информатики. 
поверхностном слое происходит перераспределение остаточных напряжений. Следует отметить, что в случае цилиндрических деталей метод опережающего упрочнения поверхностей может приводить к большему эффекту, чем непосредственное упрочнение концентратора.

Величина остаточных напряжений в наименьшем сечении цилиндрических деталей с концентраторами непосредственно связана с сопротивлением многоцикловой усталости таких изделий, поэтому основной задачей является определение остаточных напряжений в области концентратора. Решению этой задачи посвящён цикл работ В. Ф. Павлова с соавторами [1-5 и др.], методика которых базируется на расчёте дополнительных остаточных напряжений в рамках теории упругости, возникающих в наименьшем сечении детали после нанесения надреза на упрочнённую поверхность. Основные расчётные соотношения (см., например, $[2,4])$, которые использовались для определения дополнительных остаточных напряжений цилиндрических образцов диаметром 25 мм и более, получены методами теории функции комплексного переменного (ТФКП) для осевого сечения детали с надрезом [1]. Для образцов с диаметром меньше 25 мм использовалась методика, основанная на методе конечных элементов (МКЭ) и изложенная в [6]. Исходными данными для расчёта дополнительных напряжений в обоих случаях являлись значения осевой компоненты остаточных напряжений гладкой детали в области сжатия, которые определялись экспериментально. Суммируя дополнительные остаточные напряжения с напряжениями гладкой детали, авторы работ [1-5] получали остаточные напряжения детали с надрезом. Ключевым моментом этой методики является вычисление величины среднеинтегрального осевого остаточного напряжения по толщине упрочнённого слоя, опосредованно зависящей от критической глубины нераспространяющейся трещины. На основе этой методики установлены зависимости для между остаточными напряжениями и сопротивлением многоцикловой усталости для ряда деталей с концентраторами, в том числе и для авиационных резьбовых деталей [2].

В работе [7] авторами настоящей работы предложен альтернативный метод решения этой задачи на основе MKЭ в вычислительном комплексе ANSYS, учитывающий реальное распределение полей остаточных напряжений и пластических деформаций в цилиндрическом образце, который подвергся упрочнению. Исходной информацией при этом является реальное неоднородное по радиусу распределение остаточных пластических (начальных) деформаций, полученное на основе аналитического решения [8] по экспериментальной информации о распределении одной и/или двух компонент остаточных напряжений в упрочнённом слое. Начальные остаточные пластические деформации в образце с надрезом моделируются псевдотемпературными деформациями: полученное поле деформаций для гладкого цилиндрического образца переносится на образец с надрезом. После задания начальных деформаций решается задача фиктивной термоупругости относительно неизвестных остаточных напряжений. Отметим, что распределение полей остаточных напряжений для сплошного цилиндрического образца, полученное МКЭ по псевдотемпературным деформациям, практически совпадает с распределением, полученным на основе аналитического решения [8], что подтверждает корректность решения поставленной задачи. В работе [8] разработанная методика расчёта была апробирована на образцах из стали 45 и сплава Д16Т, подвергшихся 
гидродробеструйной обработке (ГДО, изотропное упрочнение, когда осевая и окружная компонента остаточных напряжений в области сжатия близки между собой, а параметр анизотропии упрочнения $\alpha=1$, см. подробнее [8,9]). Проведено сравнение результатов расчётов, выполненных для сплошных цилиндрических образцов и образцов с концентраторами, с экспериментами и расчётами, выполненными по методике [4].

В настоящей работе предложенная методика [8] применяется как для образцов (рис. 1), предварительное упрочнение которых проводилось методами изотропного упрочнения, так и для образцов с предварительным анизотропным упрочнением (обкатка роликом, алмазное выглаживание, параметр анизотропии упрочнения $\alpha \neq 1)$. При этом исследовалось влияние размера выреза полукруглого профиля на перераспределение остаточных напряжений гладкого цилиндрического образца после предварительного упрочнения поверхности, поэтому в расчётах в качестве концентраторов напряжений использовались надрезы с различным радиусом $\rho$.

Типичные картины распределения остаточных напряжений по глубине упрочнённого слоя для сплошных цилиндрических образцов различного радиуса $R$ представлены на рис. 2. Здесь значки - экспериментальные значения компонент остаточных напряжений [4], на основании которых строится аналитическое решение; сплошные линии - значения компонент остаточных напряжений, полученные по авторской методике MKЭ (отметим ещё раз, что они практически совпадают со значениями, полученными по аналитическому решению [8]).

На рис. 3 приведены распределения остаточных напряжений от дна надреза по толщине слоя в наименьшем сечении образца с концентратором напряжений в виде кругового надреза. Здесь сплошные линии - расчёт по авторской методике МКЭ для $\rho=0,3$ мм; штрихпунктирные линии - расчёт по авторской методике МКӨ для $\rho=0,5$ мм.

Для сопоставимости результатов выполнен сравнительный анализ расчётных значений для напряжения $\sigma_{z}$ по методике настоящей работы с данными расчёта этой компоненты по приближённой аналитической зависимости, полученной в [1] методами ТФКП для плоской задачи, когда вместо всего цилиндрического образца рассматривалась тонкая пластина - осевое сечение цилиндра с концентратором напряжений. На рис. 3 данные расчёта величины $\sigma_{z}$ при $\rho=0,3$ мм по методике $[1,4]$ приведены штриховой линией, при этом наблюдается соответствие данных расчёта по обеим методикам.

Одной из характерных точек эпюры остаточных напряжений является значение на поверхности. Обозначим значение компоненты $\sigma_{z}$ на поверхности цилиндрического образца через $\sigma_{\Pi z}$, а через $\sigma_{\mathrm{k} z}$ - значение компоненты $\sigma_{z}$ на поверхности концентратора напряжений. Аналогично для компонен-

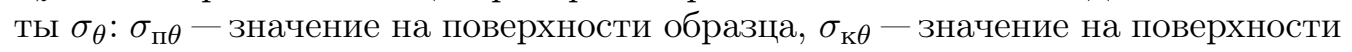

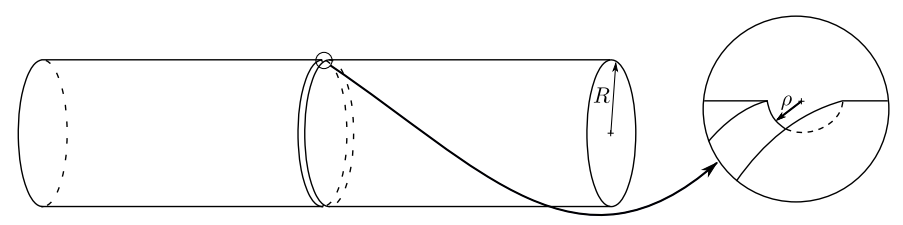

Рис. 1. Схема образца с круговым надрезом 

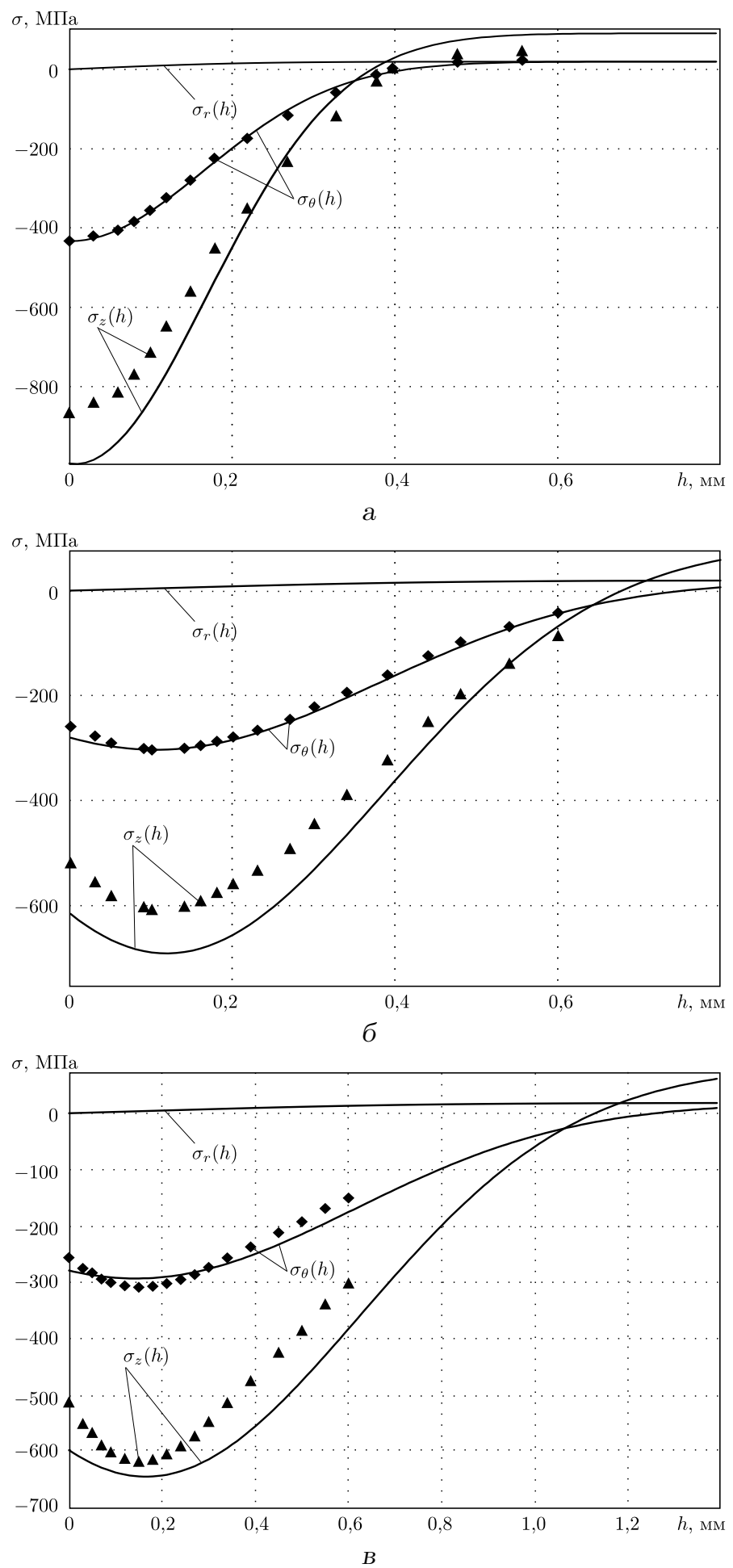

Рис. 2. Распределение остаточных напряжений по глубине $h$ упрочнённого слоя: а) сплав

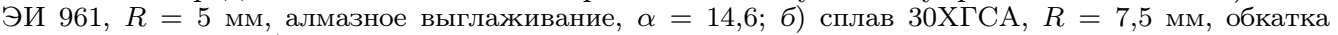
роликом, $\alpha=16,6$; в) сталь $40 \mathrm{X}, R=12,5$ мм, обкатка роликом, $\alpha=13$; сплошная линия-расчёт; значки - экспериментальные данные [4] 

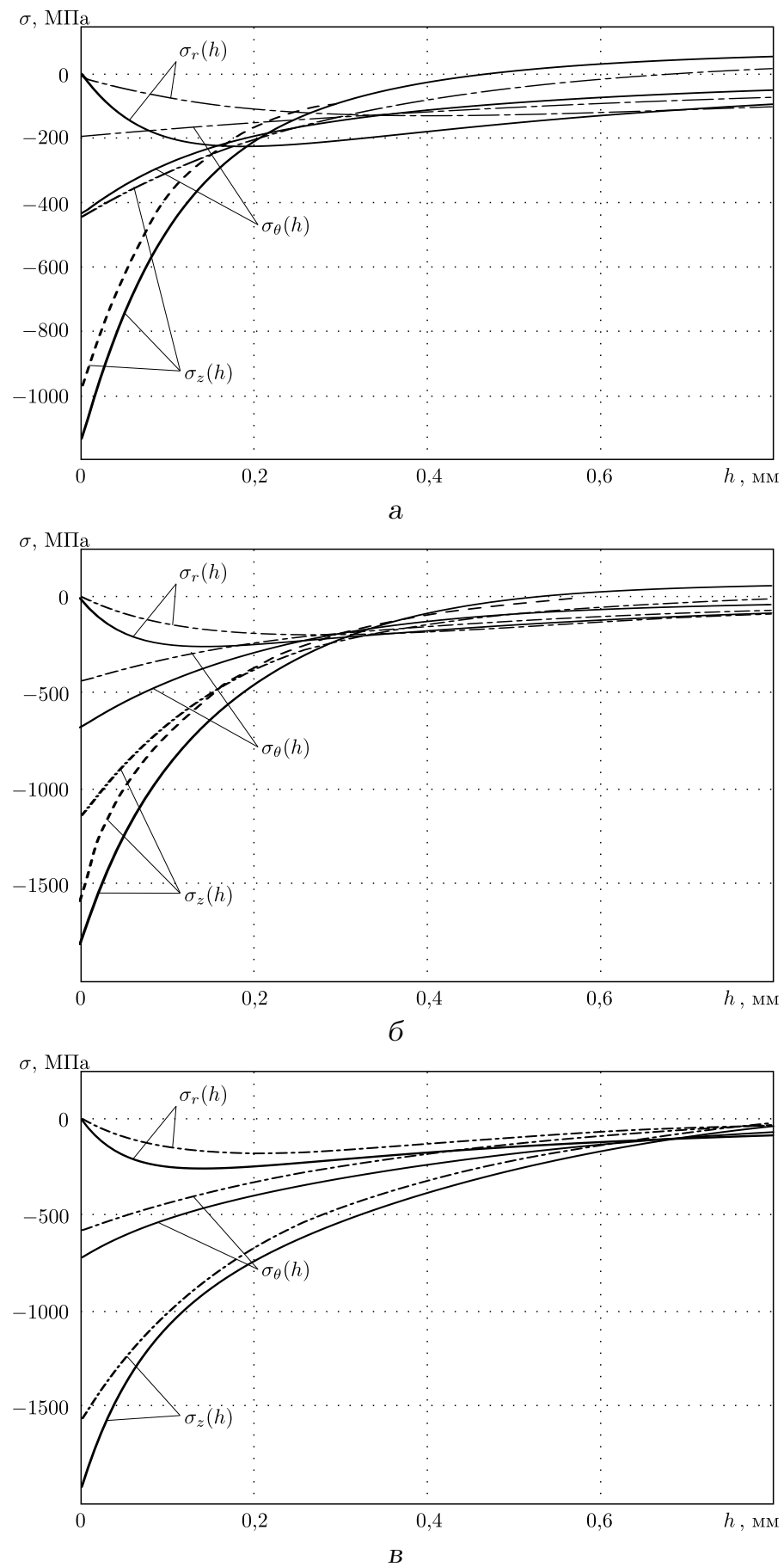

Рис. 3. Распределение остаточных напряжений от дна надреза по толщине слоя $h$ в наименьшем сечении детали: а) сплав ЭИ 961, $R=5$ мм, алмазное выглаживание, $\alpha=14,6$; б) сплав 30ХГСА, $R=7,5$ мм, обкатка роликом, $\alpha=16,6$; в) сталь $40 \mathrm{X}, R=12,5$ мм, обкатка роликом, $\alpha=13$; сплошная линия - надрез $\rho=0,3$ мм; штрихпунктирная линия - надрез $\rho=0,5$ мм; штриховая линия - расчёт по методике $[1,4]$ 
концентратора. В таблице представлены результаты расчётов для 16-ти различных цилиндрических образцов из 6-ти сплавов, подвергшихся различной технологии предварительного упрочнения, с различными концентраторами напряжений (экспериментальные данные брались из [4]). Здесь $h_{0}$ - глубина обнуления эпюры $\sigma_{z}$ для гладкого цилиндрического образца (ещё одна характерная точка).

На основании представленных данных можно сделать выводы, которые отчасти повторяют и в то же время дополняют выводы, сформулированные в работе [7]. На перераспределение остаточных напряжений в области концентратора существенное влияние оказывает отношение $\rho / h_{0}$ : увеличение $\rho / h_{0}$ приводит к уменьшению величины сжимающих остаточных напряжений $\sigma_{\theta}$ и $\sigma_{z}$ на дне концентратора, и наоборот. Так, при относительно мелких надрезах $\left(0,2<\rho / h_{0}<0,7\right)$ для исследованных образцов происходит существенное (в два и более раз) увеличение величины сжимающих остаточных напряжений $\sigma_{\theta}$ и $\sigma_{z}$ на дне концентратора по сравнению с аналогичными на-

\begin{tabular}{|c|c|c|c|c|c|c|c|}
\hline Материал & Обработка & $R, \mathrm{MM}$ & $\alpha$ & $\rho, \mathrm{MM}$ & $\rho / h 0$ & $\sigma_{\mathrm{K} z} / \sigma_{\Pi z}$ & $\sigma_{\mathrm{K} \theta} / \sigma_{\mathrm{\Pi} \theta}$ \\
\hline $12 \mathrm{X} 18 \mathrm{H} 10 \mathrm{~T}$ & ГДО & 7,50 & 1,00 & 0,30 & 0,73 & 1,84 & 0,80 \\
\hline-1 & $-1 /-$ & $-1 /-$ & $-1 /-$ & 0,50 & 1,21 & 0,84 & 0,32 \\
\hline Д16Т & ГДО & 5,00 & 1,00 & 0,30 & 0,64 & 1,42 & 0,71 \\
\hline-11 & $-1 /-$ & $-1 /-$ & $-1 /-$ & 0,50 & 1,06 & 0,65 & 0,26 \\
\hline$-1 /-$ & $-1 /-$ & 7,50 & 1,00 & 0,30 & 0,58 & 1,42 & 0,75 \\
\hline$-1 /-$ & $-1 /-$ & $-1 /-$ & $-1 /-$ & 0,50 & 0,97 & 0,70 & 0,28 \\
\hline-1 & $-1 /-$ & 12,50 & 1,00 & 0,30 & 0,51 & 1,68 & 0,80 \\
\hline-1 & $-/ /-$ & & $-/ /-$ & 0,50 & 0,85 & 0,81 & 0,32 \\
\hline $1-$ & $-1 /-$ & 20,00 & 1,00 & 0,30 & 0,47 & 1,72 & 0,83 \\
\hline$-1 /-$ & $-1 /-$ & $-1 /-$ & $-1 /-$ & 0,50 & 0,77 & 0,85 & 0,34 \\
\hline Сталь 45 & ГДО & 5,00 & 1,00 & 0,30 & 0,64 & 2,37 & 1,11 \\
\hline$-/ /-$ & $-1 /-$ & $-1 /-$ & $-1 /-$ & 0,50 & 1,06 & 0,86 & 0,34 \\
\hline$-1 /-$ & $-1 /-$ & 7,50 & 1,00 & 0,10 & 0,19 & 3,36 & 1,81 \\
\hline$-1 /-$ & $-1 /-$ & $-1 /-$ & $-1 /-$ & 0,15 & 0,29 & 2,80 & 1,64 \\
\hline$-1 /-$ & $-1 /-$ & $-1 /-$ & $-1 /-$ & 0,30 & 0,58 & 1,74 & 0,86 \\
\hline$-1 /-$ & $-1 /-$ & $-1 /-$ & $-1 /-$ & 0,50 & 0,96 & 0,94 & 0,36 \\
\hline-1 & $-1 /-$ & 12,50 & 1,00 & 0,30 & 0,55 & 2,49 & 1,20 \\
\hline$-1 /-$ & $-1 /-$ & $-1 /-$ & $-1 /-$ & 0,50 & 0,91 & 1,13 & 0,45 \\
\hline$-1 /-$ & $-1 /-$ & 25,00 & 1,00 & 0,30 & 0,50 & 2,44 & 1,18 \\
\hline$-/ /-$ & $-1 /-$ & $-1 /-$ & $-1 /-$ & 0,50 & 0,83 & 1,13 & 0,43 \\
\hline Сталь 40X & ГДО & 12,50 & 1,00 & 0,30 & 0,68 & 1,95 & 0,83 \\
\hline$-/ /-$ & $-/ /-$ & & $-/ /-$ & 0,50 & 1,13 & 0,91 & 0,33 \\
\hline 30ХГСА & обкатка роликом & 5,00 & 35,00 & 0,30 & 0,48 & 3,15 & 2,43 \\
\hline$-/ /-$ & $-/ /-$ & $-1 /-$ & $-1 /-$ & 0,50 & 0,79 & 1,68 & 1,44 \\
\hline$-1 /-$ & $-1 /-$ & 7,50 & 16,60 & 0,30 & 0,40 & 2,94 & 2,43 \\
\hline$-1 /-$ & $-1 /-$ & $-1 /-$ & $-/ /-$ & 0,50 & 0,66 & 1,84 & 1,56 \\
\hline$-1 /-$ & $-1 /-$ & 7,50 & 10,60 & 0,30 & 0,67 & 2,18 & 1,76 \\
\hline$-1 /-$ & $-/ /-$ & $-1 /-$ & $-1 /-$ & 0,50 & 1,11 & 0,88 & 0,76 \\
\hline $12 \mathrm{X} 18 \mathrm{H} 10 \mathrm{~T}$ & обкатка роликом & 5,00 & 8,20 & 0,30 & 0,66 & 2,34 & 1,77 \\
\hline$-/ /-$ & $-/ /-$ & $-/ /-$ & $-/ /-$ & 0,50 & 1,10 & 0,92 & 0,75 \\
\hline ЭИ 961 & алмазное выглаживание & 5,00 & 14,60 & 0,30 & 0,71 & 1,14 & 1,00 \\
\hline$-/ /-$ & $-/ /-$ & $-1 /-$ & $-/ /-$ & 0,50 & 1,19 & 0,45 & 0,46 \\
\hline Сталь 40X & обкатка роликом & 12,50 & 13,00 & 0,30 & 0,24 & 3,19 & 2,58 \\
\hline$-/ /-$ & $-/ /-$ & $-/ /-$ & $-/ /-$ & 0,50 & 0,40 & 2,60 & 2,17 \\
\hline
\end{tabular}


пряжениями для образцов без концентраторов напряжений. При надрезах, когда $0,75<\rho / h_{0}<1,25$, величины сжимающих остаточных напряжений $\sigma_{\theta}$ и $\sigma_{z}$ на дне концентратора становятся близкими к аналогичным напряжениям в образце без концентратора. При бо́льших значениях величины $\rho / h_{0}$ остаточные напряжения на дне концентратора становятся меньше, чем в образце без концентратора. Для величины $\sigma_{r}$ в образце с концентратором характерна смена знака, при этом её значения возрастают (по модулю) на 1-2 порядка, а увеличение $\rho / h_{0}$ приводит к уменьшению (по модулю) её значений. Радиусы цилиндрических образцов практически не оказывают влияния на указанную закономерность.

\section{БИБЛИОГРАФИЧЕСКИЙ СПИСОК}

1. Иванов С.И., Шатунов М. П., Павлов В.Ф. Определение дополнительных остаточных напряжений в надрезах на цилиндрических деталях / В сб.: Вопросы прочности элементов конструкиий: Тр. Куйбышевского авиационного института. Вып. 60. Куйбышев: КуАИ, 193. С. 160-170. [Ivanov S.I., Shatunov M. P., Pavlov V.F. Determination of the additional residual stresses in notches on cylindrical parts / In: Questions of the Strength of Elements of Aircraft Structures. Issue 60. Kuibyshev: KuAI, 193. Pp. 160-170].

2. Иванов С.И., Павлов В.Ф., Коновалов Г.В., Минин Б.В. Технологические остаточные напряжения и сопротивление усталости авиационных резьбовых деталей. М., 1992. 192 с. (Отраслевая библиотека) [Ivanov S.I., Pavlov V.F., Konovalov G.V., Minin B. V. Technological Residual Stresses and Fatigue Resistance of the Aircraft's Threaded Components. Moscow, 1992. 192 pp.]

3. Павлов В.Ф, Бордаков С.А., Сургутанова Ю.Н., Каранаева О.В., Хибник Т.А. Прогнозирование предела выносливости по разрушению деталей, изготовленных методами опережающего поверхностного пластического деформирования // Becmн. Caм. гос. техн. ун-та. Сер. Физ.-мат. науки, 2005. №34. C. 60-67. [Pavlov V. F., Bordakov S. A., Surgutanova Yu.N., Karanaeva O.V., Khibnik T.A. Prediction endurance limits for destruction of details made outstripping methods of surface plastic deformation // Vestn. Samar. Gos. Tekhn. Univ. Ser. Fiz.-Mat. Nauki, 2005. no. 34. Pp. 60-67].

4. Павлов В.Ф., Кирпичев В.А., Иванов В.Б. Остаточные напряжения и сопротивление усталости упрочненных деталей с концентраторами напряжений. Самара: Сам. науч. центр PAH, 2008. 64 с. [Pavlov V.F., Kirpichev V. A., Ivanov V. B. Residual Stresses and Fatigue Strength of Hardened Specimens with Stress Concentrators. Samara: Sam. Nauchn. Center RAN., 2008. 64 pp.]

5. Павлов В.Ф., Кирпичёв В.А., Чирков А.В., Семёнова О. Ю. Закономерности распределения дополнительных остаточных напряжений в упрочнённых цилиндрических деталях с кольцевыми надрезами полукруглого профиля// Вестн. Сам. гос. техн. унma. Сер. Физ.-мат. науки, 2010. №1(20). С. 121-126. [Pavlov V.F., Kirpichev V. A., Chirkov A. V., Semyonova O. Yu. Regularity of Auxiliary Residual Stresses Distribution into the Surface Treated Cylindrical Details with Annular Semicircular Notches // Vestn. Samar. Gos. Tekhn. Univ. Ser. Fiz.-Mat. Nauki, 2010. no. 1(20). Pp. 121-126].

6. Филатов А.П. Дополнительные остаточные напряжения на дне периодического концентратора, вызванные перераспределением остаточных напряжений гладкой детали. Куйбышев, 1985. 24 с. (Деп. в ВИНИТИ 1985. № 5157-в-85.) [Philatov A. P. Additional residual stresses on periodic stress concentrator bottom due to residual stresses on smooth part redistribution. Kuibyshev, 1985. 24 pp. (Deposited at VINITI 1984. No. 5157-v-85)]

7. Саушкин М.Н., Куров А.Ю. Конечно-элементное моделирование распределения остаточных напряжений в сплошных упроченных цилиндрических образцах и образцах с полукруглым надрезом // Вестн. Сам. гос. техн. ун-та. Сер. Физ.-мат. науки, 2011. № 3(24). C. 72-78. [Saushkin M. N., Kurov A. Yu. Finite element modeling of residual stress distribution in solid hardened cylindrical samples and samples with semicircular notch // Vestn. Samar. Gos. Tekhn. Univ. Ser. Fiz.-Mat. Nauki, 2011. no. 3(24). Pp. 72-78]. 
8. Саушкин М.Н., Радченко В. П., Павлов В.Ф. Метод расчёта полей остаточных напряжений и пластических деформаций в цилиндрических образцах с учётом анизотропии процесса поверхностного упрочнения // ПМТФ, 2011. Т. 52, № 2. С. 173-182; англ. пер.: Saushkin M.N., Radchenko V.P., Pavlov V.F. Method of calculating the fields of residual stresses and plastic strains in cylindrical specimens with allowance for surface hardening anisotropy // J. Appl. Mech. Tech. Phys., 2011. Vol.52, no. 2. Pp. 303-310.

9. Саушкин М.Н., Афанасъева О.С., Дубовова Е.В., Просвиркина Е.А. Схема расчёта полей остаточных напряжений в цилиндрическом образце с учётом организации процесса поверхностного пластического деформирования// Вестн. Сам. гос. техн. унma. Сер. Физ.-мат. науки, 2008. №1(16). C. 85-89. [Saushkin M. N., Afanas'eva O.S., Dubovova E. V., Prosvirkina E. A. The scheme calculation fields of residual stresses in the cylindrical sample when the process of surface plastic deformation into account taken // Vestn. Samar. Gos. Tekhn. Univ. Ser. Fiz.-Mat. Nauki, 2008. no. 1(16). Pp. 85-89].

Поступила в редакцию 18/I/2012;

в окончательном варианте - 29/II/2012.

MSC: 74G70; 74S05

\title{
ANALYSIS OF STRESS STATE IN SEMICIRCULAR PROFILE NOTCHES AFTER PRELIMINARY SURFACE PLASTIC DEFORMATION OF SOLID CYLINDRICAL SPECIMENS
}

\author{
M. N. Saushkin, A. Yu. Kurov
}

Samara State Technical University,

244, Molodogvardeyskaya st., Samara, 443100, Russia.

E-mails: msaushkin@gmail.com, alexeykurov@gmail.com

The distribution of residual stresses of surface hardened solid cylindrical specimens and specimens with a semicircular notch using the finite element method is studied. As the initial information the experimentally determined one and / or two components of residual stresses in a hardened layer are used. It is noted that residual stresses redistribution on the stress concentrator is significantly affected by the ratio of a notch radius to a zero depth of axial component of residual stresses.

Key words: residual stresses distribution, cylindrical specimen, semicircular notch, finite element method.

Original article submitted 18/I/2012;

revision submitted $29 / \mathrm{II} / 2012$.

Mikhail N. Saushkin (Ph. D. (Phys. \& Math.)), Doctoral Candidate, Dept. of Applied Mathematics \& Computer Science. Alexey Yu. Kurov, Student, Dept. of Applied Mathematics \& Computer Science. 